\title{
Damage Spreading in the One-Dimensional Hinrichsen-Domany Model
}

\author{
Everaldo Arashiro and J. R. Drugowich de Felício \\ Departamento de Física e Matemática \\ Faculdade de Filosofia, Ciências e Letras de Ribeirão Preto \\ Universidade de São Paulo \\ Av. dos Bandeirantes, 3900 - 14040-901 - Ribeirão Preto, SP, Brasil
}

Received on 1 November, 2000

\begin{abstract}
In this paper we study damage spreading in a one-dimensional model under two dynamics introduced by Hinrichsen and Domany. In particular, we study the effects of synchronous and asynchronous updating on the spreading properties. We show that the damage does not spread when the second dynamic is implemented in a synchronous way. We find that the rules for updating the damage produced by this dynamics, as the temperature goes to infinity and a certain parameter $\lambda$ is zero, are equivalent to those of Grassberger's well-known model A cellular automaton.
\end{abstract}

\section{Introduction}

The damage spreading technique was proposed by Kauffman thirty years ago in the context of biologically motivated cellular automata [1]. More recently, this concept was extended to analyse kinetic Ising and Potts models which are described by well-known Hamiltonians and exhibit critical properties [2]. The technique requires following the behavior of two similar samples (slightly different initial conditions) under same thermal noise. Usually, the evolution is done by means of Glauber, Metropolis or heat-bath updating. However, in order to understand the role of the dynamics, other kinds of updating have been studied. In fact, this was the goal of a recent paper by Hinrichsen and Domany [3] which describes the spreading properties in a onedimensional model submitted to two kinds of updating (henceforth named I and II). The first case (dynamics I) is very similar to Glauber dynamics but depends on the sign of the two neighbours instead of the site itself. The new value of the variable at the site $i$ is:

$\sigma_{i}(t+1)= \begin{cases}+\operatorname{sign}\left(q_{i}(t)-z\right) & \text { if } \sigma_{i-1} \sigma_{i+1}=+1 \\ -\operatorname{sign}\left(1-q_{i}(t)-z\right) & \text { if } \sigma_{i-1} \sigma_{i+1}=-1\end{cases}$

where $\sigma_{i}(t)= \pm 1, \quad z$ is a random number (between zero and 1 ) and the probability $q_{i}(t)$ is given by

$$
q_{i}(t)=\frac{e^{h_{i}(t)}}{e^{h_{i}(t)}+e^{-h_{i}(t)}}
$$

with

$$
h_{i}(t)=\frac{J}{k T} \sum_{j} \sigma_{j}(t)
$$

and $J$ the exchange coupling constant $(H=$ $\left.-J \sum_{<i, j>} \sigma_{i} \sigma_{j}\right)$. Dynamics II combines the Glauber updating with information about three sites (two first neighbours and the site itself). In this case, the new value for $\sigma$ is obtained by

$\sigma_{i}(t+1)= \begin{cases}+\operatorname{sign}\left(q_{i}(t)-z\right) & \text { if } \sigma_{i-1} \sigma_{i} \sigma_{i+1}=+1 \\ -\operatorname{sign}\left(1-q_{i}(t)-z\right) & \text { if } \sigma_{i-1} \sigma_{i} \sigma_{i+1}=-1\end{cases}$

if a random number $r$ is greater than a given parameter $\lambda$. If $r<\lambda$, the update follows Glauber dynamics

$$
\sigma_{i}(t+1)= \begin{cases}+\operatorname{sign}\left(q_{i}(t)-z\right) & \text { if } \sigma_{i}(t)=+1 \\ -\operatorname{sign}\left(1-q_{i}(t)-z\right) & \text { if } \sigma_{i}(t)=-1\end{cases}
$$

The two rules above can be combined in one updating, by introducing another parameter, $y$. Thus,

$$
\sigma_{i}(t+1)= \begin{cases}+\operatorname{sign}\left(q_{i}(t)-z\right) & \text { if } y_{i}=+1 \\ -\operatorname{sign}\left(1-q_{i}(t)-z\right) & \text { if } y_{i}=-1\end{cases}
$$

where

$$
y=\sigma_{i}\left[\left(1+\sigma_{i-1} \sigma_{i+1}\right)+\left(1-\sigma_{i-1} \sigma_{i+1}\right) \operatorname{sign}(\lambda-\check{z})\right],
$$

$\check{\mathrm{z}}$ is a random number between 0 and 1 . In both cases there is a damage spreading transition, but the critical properties are totally different. Whereas the transition in case I belongs to the directed percolation universality 
class, dynamics II leads to a "parity-conserving" phase transition. In the first case, the evolution of the damage (difference between two samples) can be described by Domany-Kinzel cellular automata rules, at least when $T=\infty$ [3]. Such a mapping is not known for the second case. In this paper we address two questions: first, What is the effect of synchronization on the spreading of the damage; second, Is there a known model that dynamics II can be mapped to?

\section{The role of updating}

To implement the dynamics presented above, we have at least two possibilities: updating one site at a time (asynchronous or "continuous dynamics") as is usual in simulation of Ising-like models, or changing all the sites at the same time (parallel or synchronous updating), usually done in cellular automata. Our simulations for dynamics I show that damage spreading occurs in both cases. We checked this conclusion by simulating the Domany-Kinzel cellular automaton (related to dynamics I, at $T=\infty$, via a mapping found by Hinrichsen and Domany) with continuous dynamics. Evolution of the damage is the same in both cases (see Fig. 1). However, for dynamics II, the situation is completely different. Depending on the kind of the updating (synchronous or asynchronous) damage spreading may or may not occur (see Fig. 2). In their study Hinrichsen and Domany [3] used only parallel updating[4]. If they had used continuous dynamics they would not have observed damage spreading, as we have shown in our simulations. In the next section, we will obtain a mapping of dynamics II to the model A cellular automaton of Grassberger et al.[5]. This will allow a better understanding of our results on damage spreading.

\section{Mapping to Grassberger's model A}

We begin by setting $\lambda=0$ (which implies working only with the equation (3.a) for updating the spins) and taking high temperature limit $(T \rightarrow \infty)$. In this case, equation (3.a) becomes

$$
\sigma_{i}(t+1)=\sigma_{i-1} \sigma_{i} \sigma_{i+1} \operatorname{sign}(0.5-z)
$$

which means that the evolution of the damage $(\Delta)$ follows deterministic rules which depend on the site itself and the two nearest neighbours:

$$
\begin{array}{ccccccccc}
t: & 111 & 110 & 101 & 100 & 011 & 010 & 001 & 000 \\
t+1: & 0 & 0 & 0 & 1 & 0 & 1 & 1 & 0
\end{array}
$$

Now we observe that these rules correspond to the one-dimensional cellular automaton obeying rule 22 (in Wolfram's classification), which is known to exhibit chaotic behavior. On the other hand, we note that the rule 22 is exactly the limit of the Grassberger's model A below with $p=1$. Thus the damage spreads.

$\begin{array}{ccccccccc}t: & 111 & 110 & 101 & 100 & 011 & 010 & 001 & 000 \\ t+1: & 0 & 1-p & 0 & 1 & 1-p & 1 & 1 & 0\end{array}$

In this sense, we can conclude that Hinrichsen-Domany's dynamics II exhibits damage spreading at least when $\lambda=0$ and $T=\infty$. On the other hand, for $\lambda=1$, and $T=\infty$, equation 4 can be simplified to

$$
\sigma_{i}(t+1)=\sigma_{i} \operatorname{sign}(0.5-z)
$$

which implies another kind of updating for the damage. We find that the local damage in this case evolves exactly as the one-dimensional cellular automaton governed by the rule 204 (again in Wolfram's notation).

$$
\begin{array}{ccccccccc} 
& \multicolumn{1}{c}{\Delta} \\
t: & 111 & 110 & 101 & 100 & 011 & 010 & 001 & 000 \\
t+1: & 1 & 1 & 0 & 0 & 1 & 1 & 0 & 0
\end{array}
$$

We remark that damage does not spread in this limit because the value of $\Delta$ is conserved in the evolution $(0 \rightarrow 0$ and $1 \rightarrow 1)$. The absence of the damage spreading in this case is not surprising, since for $\lambda=1$ we recover Glauber dynamics. 


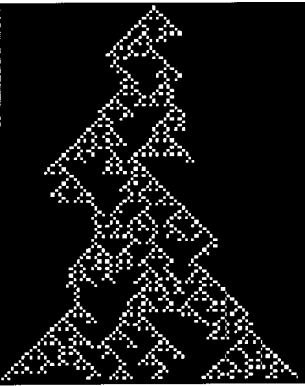

(a)

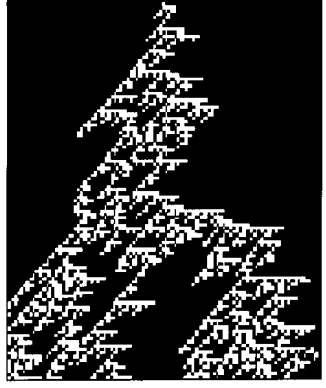

(b)
Figure 1. Patterns created with Domany-Kinzel automaton rules (with $\mathrm{p}_{1}=0.83 \mathrm{e} \mathrm{p}_{2}=0$ ). (a) Synchronous updating and (b) Asynchronous updating.

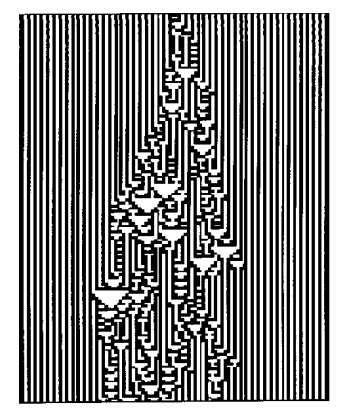

(a)

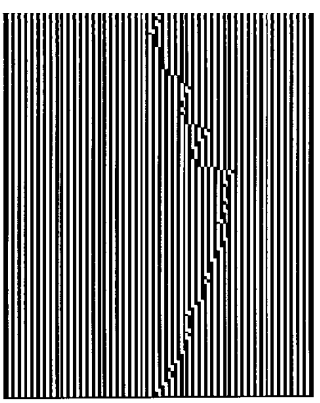

(b)
Figure 2. Patterns created with model A cellular automaton rules (with $\mathrm{p}=0.3$ ) from initial states containing a single kink. (a) Synchronous updating and (b) Asynchronous updating.

\section{Conclusions}

We studied damage spreading in a one-dimensional model evolving under two different dynamics, proposed by Hinrichsen and Domany[3]. We showed that synchronous updating can destroy the damage spreading when the dynamics II is used. In addition, we obtained a mapping of dynamics II to the model A cellular automaton introduced by Grassberger et al.[5]. In this light, it is not strange that critical exponents obtained by $\mathrm{HD}$ were in the parity conserving universality class [6].

\section{References}

[1] S. Kauffman, J. Theor. Biol. 22, 437 (1969).

[2] H.E. Stanley et al., Phys. Rev. Lett. 59, 2326 (1987)

[3] H. Hinrichsen and E. Domany, Phys. Rev. E56, 94 (1997).

[4] H. Hinrichsen, private communication.

[5] P. Grassberger, F. Krause and T. von der Twer, J. Phys. A: Math. Gen. 17, (1984).

[6] G. Ódor and N. Menyhárd, Phys. Rev. E 57, 5168 (1998). 